\title{
Time-Latitude Prominence and the Green Corona Distribution Over the Solar Activity Cycle
}

\author{
M. Minarovjech, M. Rybanský and V. Rušin \\ Astronomical Institute of the Slovak Academy of Sciences, 05960 \\ Tatranská Lomnica, Slovakia
}

\begin{abstract}
We present a distribution of prominences over the solar cycle activity. There are found both polar and equatorial branches of prominences that migrate in opposite directions. Prominences of the highlatitude crown migrate, starting in the minimum of the cycle, towards the poles, which they reach at the maximum of the cycle and then decay. The equatorward-migrating branch of prominences appears also in the minimum of the cycle at mid-latitudes and disappears at the end of the cycle. The distribution of the prominences is compared with a timelatitudinal distribution of the green corona. It is assumed that the polar branches in cycle 23 will reach the poles in 2002 (the north pole) and 2003 (the south one), respectively.
\end{abstract}

\section{Introduction}

Prominences - cool and dense material in the hot solar corona - feature not only different shapes and physical properties in a wide range but also they occur around the solar limb (Tandberg-Hanssen 1974). On a short time scale they seem to be observed above the solar limb randomly; on a long-term basis, however, we find them to be concentrated into belts, e.g., Secchi (1872), Waldmeier (1973). The structure of these belts with the solar cycle activity is, however, very complicated, especially in mid- and low-latitudes, e.g., Bumba et al. (1990), Makarov and Sivaraman (1989), Minarovjech, Rybanský and Rušin (1997). In the present work, the time-latitudinal distribution of prominences as obtained at Lomnický Śtít observatory over the period 1967 - June 1997 is studied, and subsequently compared with the distribution of the green corona $(530.3 \mathrm{~nm})$ intensity.

\section{Observations}

Observations of prominences around the whole solar limb at Lomnický Śtít have regularly been made since 1967 . A $20-\mathrm{cm}$ lens coronagraph, $\mathrm{F}=4 \mathrm{~m}$, and an $\mathrm{H} \alpha$ filter $(\mathrm{FMHW}=1.0 \mathrm{~nm}$ ) have been used. The following 5 parameters characterizing prominences are derived from these photographic observations: area (A), height $(H)$, projected width $(\mathrm{W})$, relative brightness $(\mathrm{RB})$ and heliographic position. These data have already been published for the period 1965-1986 (Rušin et al. 1988). Since 1987 the data have been available on PC diskettes. 


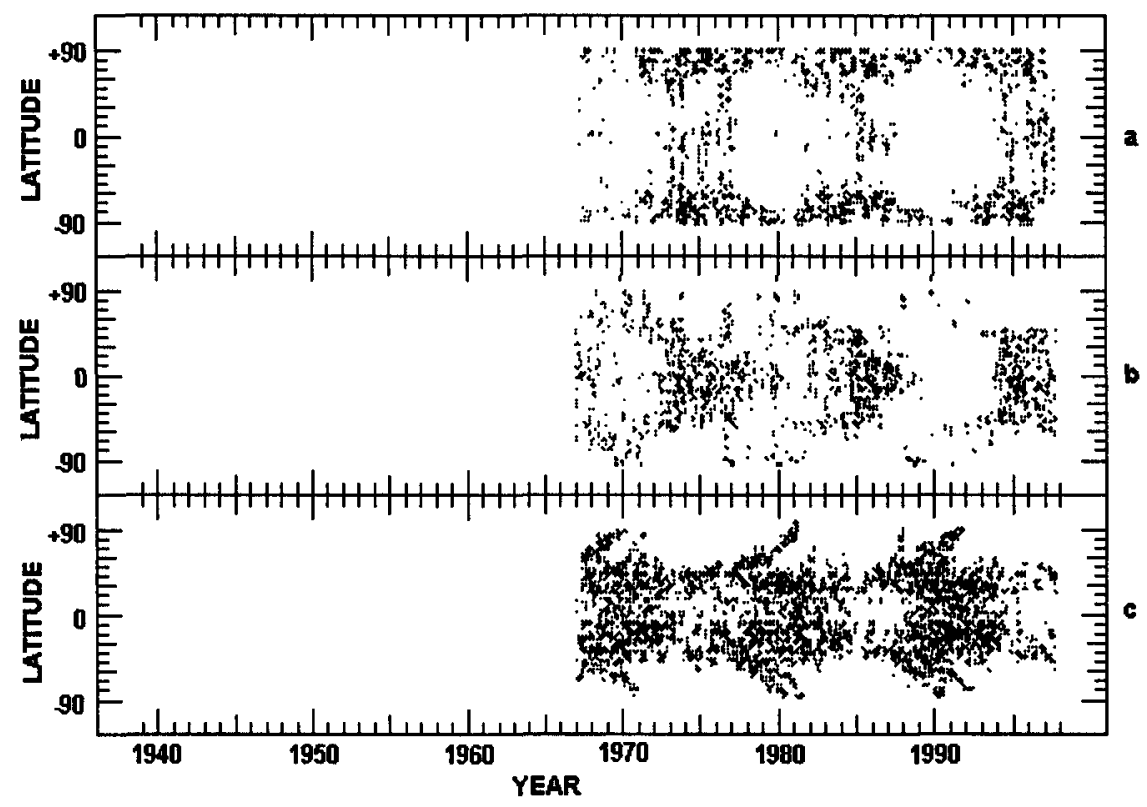

Figure 1. Time-latitudinal distribution of the averaged 'prominence index' local maxima intensities (a) values $<7$, (b) values $7-17$, (c) values $>20$.

The source for a time-latitudinal distribution of the green corona local maxima intensities are the data taken from the Homogenous Data Series (HDS) and cover the period from 1939 to 1997 (Altrock et al. 1997 and references therein). In order to find patterns, if any, in the distribution of prominences into individual belts, we divided the prominences into 3 groups according to their dependence on the value $-<7,7-17$, and $>20$ - of a new parameter, $\mathrm{PI}$, the 'prominence index'. This parameter is defined as: $R B$ (scale 1-3) $\times A$, where $A=W$ (in degrees) $\times H$ (arcsec). Computations were made for 81 -day smoothed values and in latitudinal belts of $15^{\circ}$.

\section{Discussion and Conclusion}

The corresponding time-latitudinal distribution of prominences is shown in Figure $1 \mathrm{a}$ (PI less than 7$), 1 \mathrm{~b}(\mathrm{PI}=7-17)$ and $1 \mathrm{c}(\mathrm{PI}>20)$.

One may recognize that prominences of the group a) - small and weak ones - are mainly observed around the poles of the Sun with a maximum of occurrence at latitudes of $60^{\circ}$ around the minimum of the activity cycle. These prominences disappear in about 1-2 years before the main prominences' zones (MPZ) reach their highest latitudinal distribution. They emerge again when the MPZ vanish. They move from mid-latitudes to the equator prior to the minimum, and later they show a poleward migration where they decay together with those of the type $c$ around the maximum. 
Occurrence of prominences of the group $b$ is concentrated mainly in the sunspot zone up to a latitude of $60^{\circ}$. They appear at latitudes of $60^{\circ}$ around the maximum of the activity cycle and slowly migrate toward the equator. They disappear when a polar branch of prominences is separated in mid latitudes, or shortly after this period.

Prominences in the group $c$ display a typical distribution of prominences where a formation of polar zones is clearly seen. Moreover, one sees rudiments of prominence branches of a new cycle and small 'chains' as discussed in Minarovjech et al. (1997).

Comparison between the distribution of prominences and local green coronal intensities is shown in Figure 2. Both polar coronal and prominence branches are closely connected. The local maxima of the green corona intensities reach the poles earlier than the prominence maxima. Sometimes these coronal branches are located between two polar prominence branches. Their occurrence is, however, very rare, e.g., Dermendjiev et al. (1994). Behaviour of the equatorial branches for both features of solar activity is very complicated.

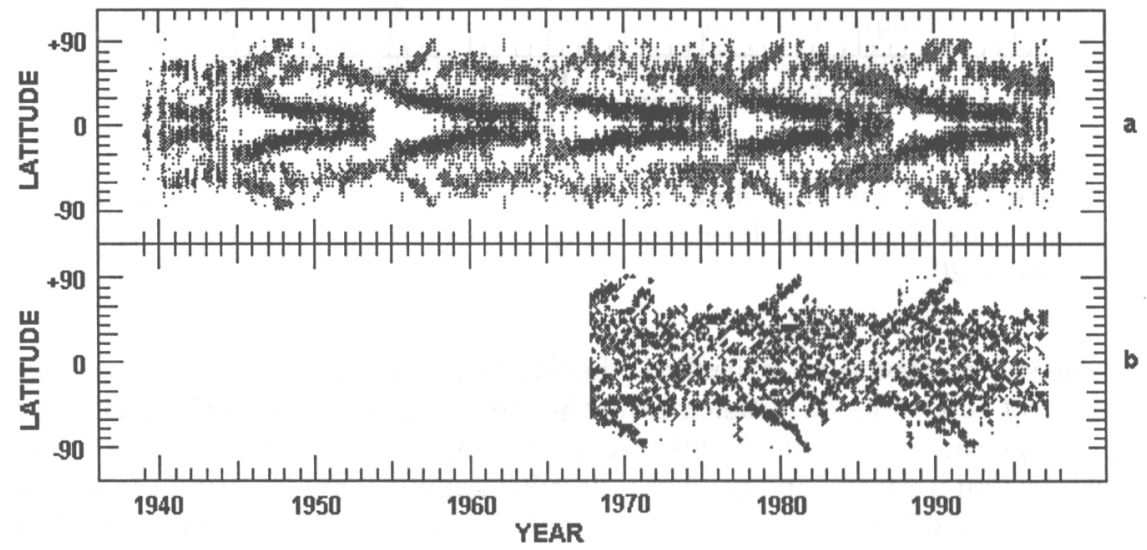

Figure 2. (a) Time-latitudinal distribution of the green corona local maxima intensities for averages of 27 days as derived from HDS, (b) Time-latitudinal distribution of the values of 'prominence index'.

Prominences, like other pronounced features of solar activity, reflect the existence of magnetic fields (local and global), which originate below the solar photosphere. However, it is well-known that the distribution of magnetic fields on the solar surface is connected with the distribution of the solar wind in the heliosphere. Long-term observations of the prominence distribution and its dynamics could help us predict some properties for the forthcoming solar cycle. Using the above data, it seems to us that a new polar branch has already separated itself from the main equatorial branch, and that this branch could reach the poles in 2002 (north) or 2003 (south).

To forecast the behaviour of both the prominence and coronal development which will occur in the polar regions in cycle 23, we start from Figure 2. When a single polar branch of prominences is observed, then two polar branches are 
observed in the green corona. The first branch (principal), which reaches the poles before the prominence branch, is brighter then the second one, occuring after the prominence branch (cycle 21). If two polar prominence branches are observed, the first one is regular, then the distribution of the green corona brightness is dim, and both coronal branches are poorly seen (cycle 20). It seems that such a pattern is observed every 22 years (the Hale cycle). Thus, we suppose that only a single branch of both corona and prominences will occur in the present cycle 23 . We note that there is a 1-year timeshift in reaching the poles by the polar prominence branches in favour of the $\mathrm{N}$-hemisphere.

The study of the corona and prominence distributions, especially in high latitudes (which are devoid of sunspots), could also help us solve a question of torsional oscillations as discovered by Howard and LaBonte (1980) and its relation to other solar activity features. As yet, these questions remain open.

Acknowledgments. This work was funded by the Grant Agency of the Slovak Academy of Sciences (Grant No. 2004/1997). One of us (M. M.) would like to thank the Organizing Committee of the IAU Colloquium 167 for financial support to attend the meeting.

\section{References}

Altrock, R. C., Rybanský, M., Rušin, V. and Minarovjech, M. 1997, Contrib. Astron. Obs. Skalnaté Pleso, 27, 25

Bumba, V. Rušin, V. and Rybanský, M. 1990, Bull. Astron. Inst. Czechosl. 41, 253

Dermendjiev, V. N., Stavrev, K. Y., Rušin, V. and Rybanský, M. 1994, A\&A, 281,241

Howard, R. and LaBonte, B. J. 1980 ApJ, 239, L33

Makarov, V. and Sivaraman, K. R. 1989, Solar Phys., 123, 367

Minarovjech, M., Rybanský, M. and Rušin, V. 1997, Solar Phys., 177, 357

Rušin, V., Rybanský, Dermendjiev, V. and Stavrev, K. Ya. 1988, Contrib. Astron. Obs. Skalnaté Pleso 17, 63

Secchi, P. A. 1872, Die Sonne, Braunschweig

Tandberg-Hanssen, E. 1974, Solar Prominences, D. Reidel, Dordrecht, Holland Waldmeier, M. 1973, Solar Phys., 28, 389 